\title{
Características de carcaça de suínos de três linhagens genéticas em diferentes idades ao abate
}

\author{
Carcass characteristics from three swine stocks in different slaughters ages
}

\author{
Alessandra Fernandes Rosa ${ }^{\mathrm{I}^{*}}$ Jacinta Diva Ferrugem Gomes $^{\mathrm{II}}$ Milena dos Reis Martelli \\ Paulo José do Amaral Sobral ${ }^{I}$ César Gonçalves de Lima ${ }^{\text {III }}$ \\ Julio César de Carvalho Balieiro ${ }^{\mathrm{III}}$
}

\section{RESUMO}

O objetivo deste trabalho foi estudar as características da carcaça de suínos de diferentes linhagens genéticas, em diferentes idades ao abate. Foram utilizados 88 suínos por linhagem, fêmeas e machos castrados, com idade $e$ peso médio iniciais de 74 dias e $30 \mathrm{~kg}$, respectivamente, pertencentes a três linhagens genéticas distintas, designadas de AgroceresPic, Dalland e Seghers. A etapa experimental foi dividida em quatro fases (Crescimento I, Crescimento II, Terminação I e Terminação II). Ao final de cada etapa, foram abatidos 60 animais (10 por linhagem/sexo), para as caracterizações: Peso (PCQ) e Rendimento de Carcaça Quente (RCQ), Área de Olho de Lombo (AOL) e Espessura de Toucinho (ET). Na análise dos resultados foi utilizado um delineamento inteiramente casualizado com desdobramento dos graus de liberdade em esquema fatorial $4 \times 3 \times 2$, sendo quatro idades ao abate (90,119, 150 e 186 dias), três linhagens (AgroceresPic, Dalland e Seghers) e dois sexos (fêmea e macho castrado), com 10 repetições por tratamento, sendo utilizado o pacote PROC MIXED do Softwear SAS. Os valores médios de peso vivo apresentaram diferença entre as linhagens e interação entre fase e linhagem $(P<0,05)$. Os valores médios das demais variáveis estudadas apresentaram diferença e a interação $(P<0,05)$ entre fase, linhagem e sexo, sendo que de maneira geral as principais diferenças ocorreram a partir da fase de Terminação I, em que as fêmeas das linhagens AgroceresPic e Dalland apresentaram melhores resultados $(P<0,05)$ de $R C Q$ (80,4 e 80,7\%, respectivamente) em comparação com os machos (78,8 e 78,7\%, respectivamente) e além disso as fêmeas Dalland apresentaram valores superiores $(P<0,01)$ de AOL $e$ ET $\left(45,7 \mathrm{~cm}^{2}\right.$ e $11,4 \mathrm{~mm} \times 38,3 \mathrm{~cm}^{2}$ e $\left.18,3 \mathrm{~mm}\right)$. Conclui-se que as linhagens genéticas avaliadas apresentaram características de carcaça muito interessantes para o mercado atual e que as fêmeas suínas podem ser utilizadas em programas que visem o abate de animais mais pesados.
Palavras-chave: suínos, rendimento de carcaça, linhagens genéticas comerciais, área de olho de lombo, espessura de toucinho.

\section{ABSTRACT}

The aim of this research was to study the carcasses characteristics of swine sire lines from different slaughter weights. In this study were used 88 castrated piglets (castrated males and females) per sire line (AgroceresPic, Dalland and Seghers), with initial age of 74 days and live weight of $30 \mathrm{~kg}$. The experiment was divided in four phases (Growing I, Growing II, Finishing I and Finishing II). Sixty animals were slaughtered (10 by stock/sex) at the end of each experimental phase to determine the post mortem measurements: Carcass Weight (CW), Hot Carcass Yield (HCW), Loin Eye Muscle Area (LMA) and Back Fat Thickness (BT). This study was conducted using a completely randomized design, with treatments in complete factorial $4 \times 3 \times 2$, being 4 slaughters ages (90, 119, 150 and 186 days), 3 sire lines (AgroceresPic, Dalland and Seghers) and 2 sexes (female and male castrated) with 10 repetitions by treatment. The PROC MIXED from the Software SAS was applied for statistical analysis. The mean values for Live Weight present difference $(P<0.05)$ sire line and interaction between phase and stock. The mean values for the other studied variables presented significant differences and interaction between phase, sire line and gender, remarking that in general the major differences occurred after the phase Finishing I, where the females of AgroceresPic and Dalland tended to present better results $(P<0.05)$ of HCW (80.4 and $80.7 \%$, respectively) than the castrated males (78.8 and $78.7 \%$, respectively) and the females Dalland were better than the castrated males $(P<0.01)$ on LMA and BT values $\left(45.7 \mathrm{~cm}^{2}\right.$ and $11,4 \mathrm{~mm} \times 38.3 \mathrm{~cm}^{2}$ and $18.3 \mathrm{~mm})$. It can be concluded that the all sire lines studied presented interesting carcass characteristics for the actual market, and that the females can be used in programs aimed at animals with more slaughter weights.

\footnotetext{
'Departamento de Engenharia de Alimentos, Faculdade de Zootecnia e Engenharia de Alimentos (FZEA), Universidade de São Paulo (USP). Av. Duque de Caxias Norte, 225, 13635-900, Pirassununga, SP, Brasil. E-mail: afrosa@usp.br.*Autor para correspondência.

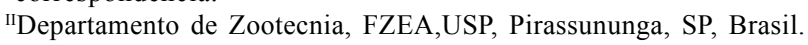

IIIDepartamento de Ciências Básicas, FZEA,USP, Pirassununga, SP, Brasil.
} 
Key words: pork, carcass yield, pork commercial lines, rib eye area, back fat thickness.

\section{INTRODUÇÃO}

$\mathrm{Na}$ suinocultura nacional atual, os programas de melhoramento genético e de plano nutricional de suínos têm enfatizado a deposição de carne magra, em detrimento à gordura, a fim de satisfazer a demanda de um mercado cada vez mais competitivo. Segundo FIALHO et al. (1998), híbridos comerciais com alto potencial genético são importantes para obtenção de progênies que produzam carcaças com menor quantidade de gordura e com maior rendimento em carne magra. A produção de suínos com carcaças mais pesadas promove algumas vantagens, tais como diminuição de custos e aumento na escala de produção, as quais são grandemente reconhecidas pela indústria frigorífica (DUTRA JR et al., 2001). Tradicionalmente, a maior limitação no aumento de peso vivo do suíno ao abate está relacionada ao aumento da gordura subcutânea em carcaças com peso superior que $100 \mathrm{~kg}$, devido principalmente à perda na eficiência alimentar, além de a possibilidade do produtor ser penalizado monetariamente pelos frigoríficos.

No entanto, o potencial genético das linhagens comerciais disponíveis no mercado vem mudando drasticamente nos últimos anos, particularmente em termos de taxas de deposição de carne magra. De acordo com CISNEROS et al. (1996), genótipos especializados em altos ganhos em carne magra têm potencial para serem abatidos mais pesados, com pouco efeito sobre a qualidade da carcaça e/ou eficiência da conversão alimentar. A quantificação do crescimento e a definição das diferenças entre sexos e linhagens são pré-requisitos essenciais para que os sistemas de integração sejam eficientes. De acordo com FÁVERO \& BELLAVER (2001), além do genótipo, o sexo também influencia o crescimento e as características de carcaça suína, sendo fator condicionante da produção de carne.

De acordo com TERRA (1998), a avaliação da carcaça é um importante indicador da sua qualidade. Assim, o objetivo deste trabalho foi avaliar as características de carcaça de suínos, fêmeas e machos castrados, de três linhagens genéticas comerciais, em diferentes idades ao abate.

\section{MATERIAL E MÉTODOS}

Esta pesquisa foi desenvolvida nas instalações do Setor de Suinocultura da Prefeitura do Campus Administrativo de Pirassununga (PCAPS), da
Universidade de São Paulo (USP). Foram utilizados como material experimental 88 suínos de cada linhagem, fêmeas e machos castrados, com 74 dias de idade e $30 \mathrm{~kg}$ de peso médio inicial, pertencentes a três linhagens genéticas distintas, designadas por AgroceresPic, Dalland e Seghers, totalizando 264 animais ao início do experimento. Os animais foram alojados em seis baias coletivas previamente sorteadas, utilizando-se 44 animais por baia, separados por linhagem e sexo. A alimentação foi formulada de acordo com as exigências nutricionais de cada linhagem genética e oferecida à vontade durante toda a etapa experimental. Após um período de adaptação de 14 dias, iniciou-se a etapa experimental, que foi dividida em quatro fases, a saber: Crescimento I - dos 74 aos 90 dias de idade, com peso vivo médio final de $45 \mathrm{~kg}$; Crescimento II - dos 91 aos 119 dias, com peso vivo médio final de $69 \mathrm{~kg}$; Terminação I - dos 120 aos 150 dias de idade, com peso vivo médio final de 96kg; e Terminação II - dos 151 aos 186 dias de idade, com peso vivo médio final de $124 \mathrm{~kg}$. Ao final de cada uma das etapas, os animais foram pesados com auxílio de uma gaiola de madeira previamente tarada, sobreposta a uma balança digital móvel com barras de ferro $(2,5 \mathrm{~kg}$, mod. MGR-2000 marca Toledo) e, 60 animais (dez por linhagem/sexo) foram abatidos, após um período de jejum de 14 a 16 horas. Os animais foram abatidos no matadouro-escola da PCAPS após insensibilização elétrica, conforme procedimento rotineiro do próprio matadouro, descrito em ROSA et al. (2001).

As carcaças quentes sem as vísceras e gordura pélvica, com cabeça e pé, foram pesadas (PCQ) com auxílio de balança de carcaça (mod. 2254L-E, marca Toledo) para o cálculo de rendimento de carcaça quente (RCQ) que foi obtido por meio da relação entre o Peso Vivo (PV) e o Peso da Carcaça Quente (PCQ) de acordo com a equação $\left\{R C Q(\%)=\frac{P V-P C Q}{P V} \times 100\right\} \mathrm{e}$, colocadas em câmara fria à temperatura de $0 \mathrm{a} 2^{\circ} \mathrm{C}$, para a instalação e resolução do rigor mortis, onde permaneceram por um período de 24 horas. Findo este período, as carcaças foram encaminhadas para a sala de desossa para determinações da Área de Olho de Lombo (AOL) e Espessura de Toucinho (ET). AAOL foi determinada de acordo com a metodologia proposta por BOGGS \& MERKEL (1979), que consistiu na secção entre a $10^{\mathrm{a}}$ e $11^{\mathrm{a}}$ costelas da meia carcaça esquerda, onde foi exposta uma seção transversal do músculo Longissimus dorsi (LD), na qual se fez decalque, em plástico transparente, do contorno do referido músculo, para posterior determinação da área com auxílio de régua quadriculada ("grid"). Esses resultados foram expressos 
em $\mathrm{cm}^{2}$. A ET foi medida diretamente na mesma seção do músculo Longissimus dorsi (LD), utilizada para determinação da AOL, perpendicularmente à pele, excluindo a mesma, com auxílio de paquímetro, expressa emmm.

$\mathrm{Na}$ análise dos resultados foi utilizado um delineamento inteiramente casualizado com desdobramento dos graus de liberdade em esquema fatorial $4 \times 3 \times 2$, sendo quatro idades ao abate $(90,119$, 150 e 186 dias), três linhagens (AgroceresPic, Dalland e Seghers) e dois sexos (fêmea e macho castrado), com 10 repetições por tratamento. Na análise dos dados de qualidade das carcaças dos suínos, utilizou-se uma abordagem de modelo com medidas repetidas (CROWDER \& HAND, 1990) em quatro fases do crescimento. O procedimento PROC MIXED do programa SAS (2000) foi utilizado nessas análises. Ocorrendo interação entre os fatores envolvidos nas análises, procedeu-se ao desdobramento de duas formas: (i) estudo das linhagens e dos sexos dentro de cada idade avaliada e (ii) estudo das idades avaliadas dentro de cada linhagem e sexo. Para comparar as médias das linhagens e sexo dentro de cada idade (i), foi utilizado o Teste $t$ de Student. Para avaliar o comportamento das idades dentro das linhagens e sexo (ii), foram utilizadas análises de regressão.

\section{RESULTADOS E DISCUSSÃO}

Observou-se interação significativa $(\mathrm{P}<0,05)$ de peso vivo dos suínos entre fase experimental e linhagem (Tabela 1), não sendo observada diferença entre os sexos. A linhagem Dalland apresentou valores médios de PV superiores nas fases Crescimento I e II. Nas fases Terminação I e II, não foi observada diferença significativa $(\mathrm{P}<0,05)$ de $\mathrm{PV}$ entre as linhagens Dalland e AgroceresPic, as quais tiveram PV superior à Seghers. O ganho diário de peso vivo das linhagens para ambos os sexos pode ser observado nos resultados das análises de regressão do peso vivo em função da idade ao abate dos animais (fase experimental) (Tabela 2).

A análise estatística dos valores médios de PCQ, RCQ, AOL e ET (Tabelas 3 e 4) permitiu a observação de diferença significativa $(\mathrm{P}<0,05)$ e interação entre idade ao abate, linhagem e sexo $(\mathrm{P}<0,05)$. Como os objetivos deste trabalho foram, em um primeiro momento, comparar os sexos da mesma linhagem e, posteriormente, comparar as linhagens do mesmo sexo, separadamente, optou-se pela apresentação e discussão dos resultados nesta sequência. Dessa forma, foi observado que os machos castrados apresentaram valores médios de PCQ superiores aos das fêmeas, sendo que essas diferenças ocorreram entre as linhagens AgroceresPic (Crescimento II) e Seghers (Terminação I e II).
Com relação ao RCQ, nas fases Crescimento I e II, os machos Dalland e AgroceresPic, respectivamente, tiveram rendimentos superiores às fêmeas. Na fase Terminação I, de maior interesse comercial, apesar de não ter sido observada diferença de PCQ entre os sexos das linhagens AgroceresPic e Dalland, as fêmeas de ambas as linhagens tiveram RCQ superior aos dos machos. Na fase Terminação II, os machos Seghers, assim como no PCQ, tiveram RCQ superiores às fêmeas.

Foi observado ainda que as fêmeas tiveram melhores resultados de AOL que os machos nas fases Crescimento I (Seghers) e Crescimento II, Terminação I e II (Dalland). Com relação à ET, os machos tiveram maiores valores, o que é indesejável, pois o mercado atual prioriza carcaças com maior quantidade de carne e menor ET. Nessas condições, as fêmeas foram superiores aos machos castrados. As diferenças ocorreram nas fases Crescimento II para as linhagens AgroceresPic e Dalland e, Terminação I e II, para Dalland e Seghers.

COSTA et al. (2005) também observaram que o efeito do sexo foi significativo para as características de peso vivo, peso de carcaça, espessura de toucinho e quantidade de gordura na carcaça, para os quais os

Tabela 1 - Valores médios e erro padrão do peso vivo dos suínos ao final de cada fase experimental, apresentados por linhagem.

\begin{tabular}{llc}
\hline & \multicolumn{2}{c}{ Peso vivo (kg) } \\
& Média* & Erro Padrão \\
\hline Crescimento I (90 dias) & & \\
AgroceresPic & $43,07^{\mathrm{b}}$ & 1,45 \\
Dalland & $46,72^{\mathrm{a}}$ & 1,45 \\
Seghers & $43,10^{\mathrm{b}}$ & 1,45 \\
Crescimento II & & \\
(119 dias) & & \\
AgroceresPic & $69,00^{\mathrm{b}}$ & 1,40 \\
Dalland & $76,52^{\mathrm{a}}$ & 1,40 \\
Seghers & $66,07^{\mathrm{c}}$ & 1,40 \\
Terminação I & & \\
(150 dias) & & 1,67 \\
AgroceresPic & $99,82^{\mathrm{a}}$ & 1,67 \\
Dalland & $100,35^{\mathrm{a}}$ & 1,67 \\
Seghers & $92,77^{\mathrm{b}}$ & \\
Terminação II & & 1,87 \\
(186 dias) & $109,73^{\mathrm{b}}$ & \\
AgroceresPic & & \\
Dalland & $129,65^{\mathrm{a}}$ & \\
Seghers & $133,26^{\mathrm{a}}$ & \\
\hline
\end{tabular}

* médias seguidas por letras minúsculas iguais não diferem entre si pelo teste t-Student $(\mathrm{P}<0,10)$.

Ciência Rural, v.38, n.6, set, 2008. 
Tabela 2 - Parâmetros das equações, calculados por regressão linear [Y $\left.=\mathrm{B}_{0}+\mathrm{B}_{1}(\mathrm{x})\right]$, em função da idade (x) por linhagem.

\begin{tabular}{|c|c|c|c|c|c|c|}
\hline $\mathrm{Y}$ & $\mathrm{B}_{0}$ & $\operatorname{Pr}>|t|$ & $\mathrm{B}_{1}(\mathrm{x})$ & $\operatorname{Pr}>|t|$ & E.P & $\mathrm{R}^{2}(\%)$ \\
\hline \multicolumn{7}{|l|}{ Peso vivo (kg) } \\
\hline AgroceresPic fêmea & $-57,37$ & $<0,0001$ & 1,05 & $<0,0001$ & 4,79 & 95,44 \\
\hline AgroceresPic macho & $-49,27$ & $<0,0001$ & 1,02 & $<0,0001$ & 5,05 & 93,66 \\
\hline Dalland fêmea & $-43,29$ & $<0,0001$ & 0,98 & $<0,0001$ & 5,22 & 92,49 \\
\hline Dalland macho & $-47,02$ & $<0,0001$ & 1,02 & $<0,0001$ & 4,91 & 94,57 \\
\hline Seghers fêmea & $-27,49$ & $<0,0001$ & 0,76 & $<0,0001$ & 4,91 & 93,60 \\
\hline Seghers macho & $-37,54$ & $<0,0001$ & 0,87 & $<0,0001$ & 4,91 & 93,25 \\
\hline \multicolumn{7}{|c|}{ Peso carcaça quente $(\mathrm{kg})$} \\
\hline AgroceresPic fêmea & $-56,39$ & $<0,0001$ & 0,91 & $<0,0001$ & 4,05 & 94,67 \\
\hline AgroceresPic macho & $-46,51$ & $<0,0001$ & 0,86 & $<0,0001$ & 4,26 & 94,72 \\
\hline Dalland fêmea & $-42,63$ & $<0,0001$ & 0,83 & $<0,0001$ & 4,40 & 93,39 \\
\hline Dalland macho & $-50,02$ & $<0,0001$ & 0,89 & $<0,0001$ & 4,15 & 95,40 \\
\hline Seghers fêmea & $-26,84$ & $<0,0001$ & 0,65 & $<0,0001$ & 4,15 & 93,23 \\
\hline Seghers macho & $-39,81$ & $<0,0001$ & 0,77 & $<0,0001$ & 4,15 & 94,08 \\
\hline \multicolumn{7}{|c|}{ Rendimento carcaça quente (\%) } \\
\hline AgroceresPic fêmea & 64,20 & $<0,0001$ & 0,11 & $<0,0001$ & 2,09 & 35,16 \\
\hline AgroceresPic macho & 66,73 & $<0,0001$ & 0,08 & $<0,0001$ & 2,10 & 74,89 \\
\hline Dalland fêmea & 66,78 & $<0,0001$ & 0,08 & $<0,0001$ & 2,10 & 58,12 \\
\hline Dalland macho & 58,55 & $<0,0001$ & 0,13 & $<0,0001$ & 2,10 & 72,38 \\
\hline Seghers fêmea & 73,25 & $<0,0001$ & 0,05 & 0,0004 & 2,10 & 23,70 \\
\hline Seghers macho & 66,29 & $<0,0001$ & 0,09 & $<0,0001$ & 2,10 & 62,99 \\
\hline \multicolumn{7}{|c|}{ Área de olho de lombo $\left(\mathrm{cm}^{2}\right)$} \\
\hline AgroceresPic fêmea & $-11,94$ & $<0,0001$ & 0,36 & $<0,0001$ & 2,78 & 85,07 \\
\hline AgroceresPic macho & $-11,28$ & 0,0003 & 0,36 & $<0,0001$ & 2,93 & 91,63 \\
\hline Dalland fêmea & $-11,66$ & 0,0003 & 0,37 & $<0,0001$ & 3,03 & 83,89 \\
\hline Dalland macho & $-0,80$ & 0,7804 & 0,26 & $<0,0001$ & 2,85 & 78,40 \\
\hline Seghers fêmea & 3,35 & 0,2577 & 0,23 & $<0,0001$ & 2,93 & 79,96 \\
\hline Seghers macho & $-1,69$ & 0,5625 & 0,27 & $<0,0001$ & 2,91 & 72,71 \\
\hline \multicolumn{7}{|c|}{ Espessura de toucinho (mm) } \\
\hline AgroceresPic fêmea & $-11,63$ & $<0,0001$ & 0,18 & $<0,0001$ & 2,79 & 56,08 \\
\hline AgroceresPic macho & $-5,25$ & 0,0808 & 0,14 & $<0,0001$ & 2,96 & 60,31 \\
\hline Dalland fêmea & $-6,69$ & 0,0335 & 0,14 & $<0,0001$ & 3,08 & 61,71 \\
\hline Dalland macho & $-16,74$ & $<0,0001$ & 0,26 & $<0,0001$ & 2,88 & 68,22 \\
\hline Seghers fêmea & $-5,38$ & 0,0550 & 0,14 & $<0,0001$ & 2,87 & 43,20 \\
\hline Seghers macho & $-16,84$ & $<0,0001$ & 0,25 & $<0,0001$ & 2,87 & 77,44 \\
\hline
\end{tabular}

machos tiveram valores médios superiores, enquanto que para rendimento de carne na carcaça as fêmeas foram superiores. Segundo esses autores, este fato pode ser parcialmente explicado em virtude dos machos castrados apresentarem maior consumo de ração em relação às fêmeas, o que implica em um maior potencial de deposição de gordura na carcaça.

Como pode ser observado ainda na tabela 2, de maneira geral, os valores de PCQ, RCQ, AOL e ET aumentaram linearmente durante o período avaliado. As fêmeas da linhagem AgroceresPic tenderam a apresentar taxas de ganhos superiores aos machos para todas as variáveis, com exceção da variável AOL, em que as taxas de ganho foram iguais. Por outro lado, os machos Dalland foram mais eficientes nos ganhos de
PCQ, RCQ e ET em relação às fêmeas, o que indica que apesar de as fêmeas Dalland apresentarem menores taxas de ganho, tanto em peso quanto em rendimento, apresentaram melhor eficiência de conversão em carne na carcaça, indicado pelos valores superiores de AOL. Isso pode se traduzir em vantagem econômica, uma vez que a AOL implica melhor rendimento de carne magra na carcaça (TERRA, 1998).

Os resultados deste trabalho corroboram os resultados de FIALHO et al. (1998), IRGANG et al. (1997) e FÁVERO \& BELLAVER (2001). Esses trabalhos observaram que as fêmeas suínas são mais eficientes que os machos castrados na transformação do alimento consumido em carne. Por outro lado, CISNEROS et al. (1996) e LATORRE et al. (2003) não observaram 
Tabela 3 - Valores médios e erro padrão das medidas de peso e rendimento de carcaça quente dos suínos apresentados por fase experimental, linhagem e sexo.

\begin{tabular}{|c|c|c|c|c|c|c|c|c|}
\hline \multirow[b]{3}{*}{ Idade/Genética } & \multicolumn{4}{|c|}{--------------'Peso carcaça quente $(\mathrm{kg})$------------- } & \multicolumn{4}{|c|}{----------Rendimento carcaça quente (\%)-------- } \\
\hline & \multicolumn{2}{|c|}{ Fêmea } & \multicolumn{2}{|c|}{ Macho } & \multicolumn{2}{|c|}{ Fêmea } & \multicolumn{2}{|c|}{ Macho } \\
\hline & Média* & $\mathrm{EP}$ & Média* & EP & Média* & $\mathrm{EP}$ & Média* & $\mathrm{EP}$ \\
\hline \multicolumn{9}{|c|}{ Crescimento I (90 dias) } \\
\hline AgroceresPic & $31,08^{\mathrm{aA}}$ & 1,62 & $33,92^{\mathrm{aA}}$ & 1,62 & $76,85^{\mathrm{aAB}}$ & 1,22 & $74,60^{\mathrm{aA}}$ & 1,22 \\
\hline Dalland & $33,92^{\mathrm{aA}}$ & 1,62 & $33,10^{\mathrm{aA}}$ & 1,62 & $74,09^{\mathrm{aB}}$ & 1,22 & $69,06^{\mathrm{bB}}$ & 1,22 \\
\hline Seghers & $34,20^{\mathrm{aA}}$ & 1,62 & $32,20^{\mathrm{aA}}$ & 1,62 & $78,12^{\mathrm{aA}}$ & 1,22 & $76,19^{\mathrm{aA}}$ & 1,22 \\
\hline \multicolumn{9}{|c|}{ Crescimento II (119 dias) } \\
\hline AgroceresPic & $49,28^{\mathrm{bB}}$ & 1,59 & $56,09^{\mathrm{aA}}$ & 1,59 & $74,34^{\mathrm{bB}}$ & 0,85 & $78,14^{\mathrm{aA}}$ & 0,85 \\
\hline Dalland & $58,14^{\mathrm{aA}}$ & 1,59 & $57,09^{\mathrm{aA}}$ & 1,59 & $75,67^{\mathrm{aB}}$ & 0,85 & $75,23^{\mathrm{aB}}$ & 0,85 \\
\hline Seghers & $49,10^{\mathrm{aB}}$ & 1,59 & $51,37^{\mathrm{aB}}$ & 1,59 & $78,04^{\mathrm{aA}}$ & 0,85 & $76,35^{\mathrm{aAB}}$ & 0,85 \\
\hline \multicolumn{9}{|c|}{ Terminação I (150 dias) } \\
\hline AgroceresPic & $79,27^{\mathrm{aA}}$ & 1,82 & $79,47^{\mathrm{aA}}$ & 1,82 & $80,39^{\mathrm{aA}}$ & 0,52 & $78,79^{\mathrm{bB}}$ & 0,52 \\
\hline Dalland & $79,47^{\mathrm{aA}}$ & 1,82 & $80,41^{\mathrm{aA}}$ & 1,82 & $80,69^{\mathrm{aA}}$ & 0,52 & $78,70^{\mathrm{bB}}$ & 0,52 \\
\hline Seghers & $71,42^{\mathrm{bB}}$ & 1,82 & $79,40^{\mathrm{aA}}$ & 1,82 & $81,31^{\mathrm{aA}}$ & 0,52 & $81,24^{\mathrm{aA}}$ & 0,52 \\
\hline \multicolumn{9}{|c|}{ Terminação II (186 dias) } \\
\hline AgroceresPic & $107,51^{\mathrm{aA}}$ & 2,02 & $108,08^{\mathrm{aA}}$ & 2,43 & $83,00^{\mathrm{aA}}$ & 0,51 & $82,11^{\mathrm{aA}}$ & 0,52 \\
\hline Dalland & $106,05^{\mathrm{aA}}$ & 2,75 & $109,69^{\mathrm{aA}}$ & 2,19 & $80,87^{\mathrm{aB}}$ & 0,52 & $81,34^{\mathrm{aB}}$ & 0,51 \\
\hline Seghers & $87,43^{\mathrm{bB}}$ & 2,19 & $94,48^{\mathrm{aB}}$ & 2,19 & $81,61^{\mathrm{bB}}$ & 0,51 & $83,04^{\mathrm{aA}}$ & 0,51 \\
\hline
\end{tabular}

* médias seguidas por letras minúsculas (linhas) e maiúsculas (colunas) iguais, na mesma fase, não diferem entre si pelo teste t-Student $(\mathrm{P}<0,10)$.

diferença de PCQ e RCQ entre os sexos, porém ambos relatam que os machos tiveram carcaças com maior quantidade de gordura que as fêmeas.

$\mathrm{O}$ estudo comparativo entre as linhagens fềmeas demonstrou que as fềmeas Dalland tiveram maior PCQ na fase Crescimento II e, nas fases subseqüentes, as linhagens AgroceresPic e Dalland foram estatisticamente iguais e superiores à Seghers. Observou-se ainda que para o RCQ as diferenças ocorreram nas fases Crescimento I e II, em que as Seghers tiveram RCQ superior e, na fase Terminação II, as AgroceresPic tiveram melhor rendimento. Com relação às medidas de $\mathrm{AOL}$, foram observadas diferenças nas fases Terminação I e II, em que as fêmeas AgroceresPic e Dalland apresentaram medidas de AOL superiores as da Seghers. Por outro lado, para a ET, só foi observada diferença na fase Terminação I, em que as fêmeas da linhagem Dalland apresentaram as menores medidas.

Entre os machos, o estudo comparativo demonstrou que para o PCQ só ocorreram diferenças nas fases Crescimento II e Terminação II, em que os machos AgroceresPic e Dalland foram estatisticamente iguais e superiores $(\mathrm{P}<0,05)$ aos Seghers. No entanto, as diferenças de RCQ podem ser observadas em todas as fases experimentais. De maneira geral, os machos Seghers e AgroceresPic foram os que tiveram os melhores rendimentos. Para as variáveis AOL e ET, as diferenças entre as linhagens só foram observadas nas fases Terminação I e II. Na fase Terminação I, os machos AgroceresPic e Seghers tiveram valores de AOL estatisticamente iguais e superiores aos Dalland, enquanto que para a ET os AgroceresPic e Dalland foram iguais e tiveram medidas inferiores aos Seghers. Na fase Terminação II, os machos AgroceresPic tiveram maior AOL e menor ET que os Dalland e Seghers, os quais foram estatisticamente iguais para as duas variáveis.

Este estudo permitiu constatar ainda que, apesar de os animais da linhagem Dalland apresentarem, na fase Terminação II, valores médios de peso vivo estatisticamente iguais aos da linhagem AgroceresPic, os RCQ foram inferiores para as fêmeas, o que sugere maior peso de conteúdo gastrintestinal. Porém, pode-se observar também um aumento considerável nos valores médios de RCQ dos 90 aos 186 dias de idade, o que demonstra a eficiência da utilização de linhagens modernas associadas ao plano nutricional adequado.

Os valores de ET e AOL determinados neste estudo também corroboram o trabalho do melhoramento genético, uma vez que, em níveis de desenvolvimento muscular bastante próximos, determinados por meio da AOL, as espessuras de toucinho reduziram significativamente, indicando uma melhor capacidade dessas linhagens em depositar tecido magro na carcaça, o que pode ser revertido em ganho na produção. Pode-se citar, como exemplo, IRGANG \& 
Tabela 4 - Valores médios e erro padrão das medidas de área de olho de lombo e espessura de toucinho de suínos apresentados por fase experimental, linhagem e sexo.

\begin{tabular}{|c|c|c|c|c|c|c|c|c|}
\hline \multirow[b]{3}{*}{ Idade/Genética } & \multicolumn{4}{|c|}{---------Área de olho de lombo $\left(\mathrm{cm}^{2}\right)$--------- } & \multicolumn{4}{|c|}{----------Espessura de toucinho $(\mathrm{mm})$--------- } \\
\hline & \multicolumn{2}{|c|}{ Fêmea } & \multicolumn{2}{|c|}{ Macho } & \multicolumn{2}{|c|}{ Fêmea } & \multicolumn{2}{|c|}{ Macho } \\
\hline & Média* & EP & Média* & EP & Média* & EP & Média* & EP \\
\hline \multicolumn{9}{|l|}{$\begin{array}{l}\text { Crescimento I } \\
\text { (90 dias) }\end{array}$} \\
\hline AgroceresPic & $20,60^{\mathrm{aA}}$ & 1,14 & $22,90^{\mathrm{aA}}$ & 1,14 & $6,16^{\mathrm{aA}}$ & 0,11 & $7,2^{\mathrm{aA}}$ & 0,11 \\
\hline Dalland & $22,15^{\mathrm{aA}}$ & 1,14 & $22,10^{\mathrm{aA}}$ & 1,14 & $6,39^{\mathrm{aA}}$ & 0,11 & $6,60^{\mathrm{aA}}$ & 0,11 \\
\hline Seghers & $24,20^{\mathrm{aA}}$ & 1,14 & $21,23^{\mathrm{bA}}$ & 1,21 & $7,72^{\mathrm{aA}}$ & 0,11 & $6,85^{\mathrm{aA}}$ & 0,11 \\
\hline \multicolumn{9}{|l|}{$\begin{array}{l}\text { Crescimento II } \\
\text { (119 dias) }\end{array}$} \\
\hline AgroceresPic & $32,30^{\mathrm{aA}}$ & 1,04 & $30,85^{\mathrm{aA}}$ & 1,04 & $8,60^{\mathrm{aA}}$ & 0,09 & $12,37^{\mathrm{bA}}$ & 0,09 \\
\hline Dalland & $33,60^{\mathrm{aA}}$ & 1,04 & $30,75^{\mathrm{bA}}$ & 1,04 & $10,58^{\mathrm{aA}}$ & 0,09 & $15,23^{\mathrm{bA}}$ & 0,10 \\
\hline Seghers & $32,15^{\mathrm{aA}}$ & 1,04 & $32,05^{\mathrm{aA}}$ & 1,04 & $11,40^{\mathrm{aA}}$ & 0,09 & $13,12^{\mathrm{aA}}$ & 0,09 \\
\hline \multicolumn{9}{|l|}{$\begin{array}{l}\text { Terminação I } \\
\text { (150 dias) }\end{array}$} \\
\hline AgroceresPic & $43,40^{\mathrm{aA}}$ & 1,23 & $42,45^{\mathrm{aA}}$ & 1,23 & $15,05^{\mathrm{aB}}$ & 0,11 & $16,60^{\mathrm{aA}}$ & 0,11 \\
\hline Dalland & $45,75^{\mathrm{aA}}$ & 1,23 & $38,30^{\mathrm{bB}}$ & 1,23 & $11,39^{\mathrm{aC}}$ & 0,11 & $18,32^{\mathrm{bA}}$ & 0,11 \\
\hline Seghers & $38,55^{\mathrm{aB}}$ & 1,23 & $41,15^{\mathrm{aAB}}$ & 1,23 & $18,05^{\mathrm{aA}}$ & 0,11 & $21,45^{\mathrm{bB}}$ & 0,11 \\
\hline \multicolumn{9}{|l|}{$\begin{array}{l}\text { Terminação II } \\
\text { (186 dias) }\end{array}$} \\
\hline AgroceresPic & $50,22^{\mathrm{aA}}$ & 1,43 & $53,29^{\mathrm{aA}}$ & 1,72 & $20,68^{\mathrm{aA}}$ & 0,14 & $18,62^{\mathrm{aA}}$ & 0,17 \\
\hline Dalland & $51,15^{\mathrm{aA}}$ & 1,95 & $43,00^{\mathrm{bB}}$ & 1,55 & $21,04^{\mathrm{aA}}$ & 0,19 & $31,12^{\mathrm{bB}}$ & 0,15 \\
\hline Seghers & $44,21^{\mathrm{aB}}$ & 1,55 & $41,15^{\mathrm{aB}}$ & 1,66 & $19,53^{\mathrm{aA}}$ & 0,16 & $27,63^{\mathrm{bB}}$ & 0,15 \\
\hline
\end{tabular}

* médias seguidas por letras minúsculas (linhas) e maiúsculas (colunas) iguais, na mesma fase, não diferem entre si pelo teste t-Student $(\mathrm{P}<0,10)$.

PROTAS (1986), que ao trabalharem com suínos de raças puras aos $80,110,120$ e $140 \mathrm{~kg}$ de peso vivo determinaram valores médios de ET da ordem 29, 32, 38 e $40 \mathrm{~mm}$, respectivamente. Posteriormente, IRGANG et al. (1998) relataram que, em $1995,87 \%$ do total das carcaças dos animais abatidos na região Sul do país tiveram ET variando de 15 a $34 \mathrm{~mm}$ com média de 23 a $24 \mathrm{~mm}$.

Os resultados deste trabalho permitiram se observar ainda que os suínos estudados apresentaram bons resultados de características de carcaça na fase Terminação II. Isso pode ser um indicativo para estudos futuros da viabilidade econômica da utilização de animais mais pesados nos atuais sistemas de produção.

\section{CONCLUSÕES}

As linhagens genéticas avaliadas apresentaram características de carcaça muito interessantes para o mercado atual. Por apresentarem melhores características de carcaças, as fêmeas suínas podem ser utilizadas em programas que visem o abate de animais mais pesados.

\section{AGRADECIMENTOS}

À FAPESP, pela bolsa de IC (02/07022-5) para MRM e de MS (01/12646-5) para AFR.

\section{REFERÊNCIAS}

BOGGS, D.L.; MERKEL, A.R. Live animal carcass evaluation and selection manual. Dubuque: Kendal/Hunt, 1979.

CISNEROS, F. et al. Influence of slaughter weight on growth and carcass characteristics, commercial cutting and curing yields, and meat quality of barrows and gilts from two genotypes. Journal of Animal Science, v.74, p.925-933, 1996.

COSTA, M.C.R. et al. Utilização da torta de girassol na alimentação de suínos nas fases de crescimento e terminação: Efeitos no desempenho e nas características de carcaça. Revista Brasileira de Zootecnia, v.34, p.1581-1588, 2005.

CROWDER, M.J.; HAND, D.J. Analysis of repeated measures. London: Chapman \& Hall, 1990. 257p.

DUTRA JR, W.M. et al. Estimativas de rendimentos de cortes comerciais e de tecidos de suínos em diferentes pesos de abate 
pela técnica de ultra-sonografia em tempo real. Revista Brasileira de Zootecnia, v.30, p.1243-1250, 2001.

FÁVERO, J.A.; BELLAVER, C. Produção de carne de suínos. In: CONGRESSO BRASILEIRO DE CIÊNCIA E TECNOLOGIA DE CARNES, 2001, São Pedro, SP. Anais... São Pedro/SP: ITAL, Instituto de Tecnologia de Alimentos, 2001. p.2-25.

FIALHO, E.T. et al. Influência de planos de nutrição sobre as características de carcaça de suínos de diferentes genótipos abatidos entre 80 e $120 \mathrm{~kg}$. Revista Brasileira de Zootecnia, v.27, p.1140-1146, 1998.

IRGANG, R.; PROTAS, J.F.S. Peso ótimo de abate de suínos. II resultados de carcaça. Pesquisa Agropecuária Brasileira, Brasília, v.21, p.337-1345, 1986.

IRGANG, R. Influência genética sobre o rendimento e a qualidade da carne de suínos. In: CONGRESSO BRASILEIRO DE VETERINÁRIOS ESPECIALISTAS EM SUÍNOS, 8., Anais... p.145-151, 1997.
IRGANG, R. et al. Medidas de espessura de toucinho e de profundidade de músculo para estimar rendimento de carne em caraças de suínos. Revista Brasileira de Zootecnia, v.27, p.928-935, 1998.

LATORRE, M.A. et al. Effect of sex and terminal sire genotype on performance, carcass characteristics, and meat quality of pigs slaughtered at $117 \mathrm{~kg}$ body weight. Meat Science, v.65, p.1369$1377,2003$.

ROSA, A.F.et al. Determinação das características físico-químicas da carne de suínos em fase de crescimento. Revista TeC Carnes, v.3, p.13-18, 2001.

SAS Institute Inc. SAS/STAT ${ }^{\circledR}$ OnlineDoc $^{\circledR}$, Version 8 , Copyright $^{\circ}$ 2000. Cary, 2000.

TERRA, N.N. Apontamentos de tecnologia de carnes. São Leopoldo: Unisinos, 1998. 216p. 